\title{
A Review on Enhanced Channel Equalizers for Adaptive Zero-Guard-Interval CO-OFDM Systems
}

\author{
Abdul Saad Khan Choudhary \\ Research Scholar in \\ Digital Communication, \\ NITTTR, Bhopal, MP, India
}

\author{
Anjali Ashish Potnis, PhD \\ Asst. Professor in DEEE, \\ NITTTR, Bhopal, MP, India
}

\begin{abstract}
In order to satisfy the ever rising demand for the bandwidth requirement in broadband services the Coherent orthogonal frequency division multiplexing (COFDM) method is being considered as a promising technique for future high-capacity optical networks. The aim of this proposed is to investigate, theoretically, the feasibility of implementing the coherent optical OFDM (CO-OOFDM) technique in extensive transmission networks. For CO-OOFDM and Fast-OFDM systems a set of modulation formats dependent analog to digital converter (ADC) cutting ratio and the quantization bit have been identified, moreover, CO-OOFDM is additionally resilient to the chromatic dispersion (CD) when compared to the bandwidth efficient Fast-OFDM scheme. Also proposed adaptive equalization and ANN to reduce overhead problem in training symbol.
\end{abstract}

\section{Keywords}

Adaptive equalizer, coherent detection, orthogonal frequency division multiplexing.

\section{INTRODUCTION}

At the present time, satisfying the wants of the end user for communication speed and bandwidth as well as offering improved and protected quality of services is the real inspiration behind the rapid increase in the internet protocol (IP) traffic and newly promising applications such as IP television (IPTV), video on demand (VoD) and video surveillance. This adds more force on the networks infrastructure at every scale, which explains the real motive behind all optical communications research. Coherent optical orthogonal frequency division multiplexing (CO-OFDM) systems are potential candidates for future software defined flexible transceiver implementation attributable to their compact spectrum, flexibility in spectral efficiency and bandwidth management, and universal digital signal processing (DSP) techniques. Coherent optical orthogonal frequency division multiplexing (CO-OFDM) offers advantages such as its dispersion tolerance ease of frequency domain equalization and high spectral efficiency. Several experiments on CO-OFDM transmission have proved it as a suitable candidate for the $100 \mathrm{~Gb} / \mathrm{s}$ Ethernet transport. OFDM is more sensitive to linear phase noise compared to a single carrier system. Furthermore, as the OFDM sign is adjust in the frequency domain, algorithms like M-th power cannot be applied in the time domain previous to Fast Fourier transform (FFT). Uncompensated phase noise will cause common phase error (CPE) and inter carrier interference (ICI) to the received signal after FFT.

Thus, a compensation method before FFT is preferred as it allows removing ICI. Coherent optical orthogonal frequency division multiplexing (CO-OFDM) has been thought of as a promising candidate for long-haul optical communication systems due to its high spectral efficiency and excellent tolerance towards linear fiber destruction, such as chromatic distribution and polarization mode dispersion. Orthogonal frequency division multiplexing (OFDM) has been utilized in many telecommunication applications due to its high spectral efficiency and simple hardware implementation. OFDM has additionally been thought of for optical systems as a candidate for future long range high data rate communication systems. The principle of frequency multiplexing is to group the digital information by $\mathrm{N}$ packets, which can be known as OFDM symbol and modulating each given a different carrier simultaneously. one of the central features that set orthogonal frequency division multiplexing (OFDM) aside from singlecarrier modulation is its uniqueness of signal processing. COOFDM system is setup by using matlab simulation for the transmitter and receiver blocks; also the channel is simulated with Split Step Fourier methodology as a solution for NLSE (Non Linear Schrödinger Equation). it's been employed by many researchers to simulate the fiber nonlinearity and dispersion effects in optical communication systems. It takes most key optical communication system/component parameters under consideration as well as fiber nonlinearity, noise, dispersion, and PMD etc..

\subsection{Coherent optical OFDM}

The principles of orthogonal frequency division multiplexing (OFDM) modulation are in existence for several decades. However, in recent years these techniques have quickly moved out of textbooks and analysis laboratories and into apply in modern communications systems. The techniques are employed in information delivery systems over the line, digital radio and TV, and wireless networking systems. the foremost important assumption for OFDM is that the onedimensionality in modulation, transmission, and reception. Consequently, a linear transformation is that the key goal for the OFDM implementation. A generic optical OFDM system will be divided into 5 purposeful blocks including (i) the RF OFDM transmitter, (ii) the RF-to-optical (RTO) up-converter, (iii) the optical channel, (iv) the optical-to-RF (OTR) downconverter, and (v) the RF OFDM receiver [1]. it's apparent that the challenges for CO-OFDM implementation are to obtain a linear RTO up-converter and linear OTR downconverter. It's been planned and analyzed that by biasing the Mach-Zehnder modulators (MZMs) at null point, a linear conversion between the RF signal and optical field signal may be achieved [1]. It's also been shown that by using coherent detection, a linear transformation from optical field signal to RF (or electrical) signal will be achieved.

\section{LITERATURE REVIEW}

[1] Wei Wang, et. al. "Enhanced Channel Equalizers for Adaptive Zero-Guard-Interval CO-OFDM Systems", demonstrated single channel ZGI CO-OFDM transmission with proposed FD-BLMS and FD-BRLS adaptive equalizers 
for $100 \mathrm{~Gb} / \mathrm{s}(\mathrm{QPSK})$ and $200 \mathrm{~Gb} / \mathrm{s}$ (16-QAM) systems and numerically studied their dynamic time tracking abilities. In the experiments, with the enhanced equalizer designs, the transmission distance is increased by $20 \%$ and $7 \%$ for 112 $\mathrm{Gb} / \mathrm{s}$ QPSK system over $6500 \mathrm{~km} \mathrm{SSMF} \mathrm{(7 \%} \mathrm{FEC),} \mathrm{and} \mathrm{53 \%}$ and $35 \%$ for $250 \mathrm{~Gb} / \mathrm{s}$ 16-QAM system over $2080 \mathrm{~km} \mathrm{SSMF}$ (20\% FEC), compared with the TS based and decision aided $\mathrm{ZF}$ adaptive equalizers, respectively.

[2] Man Pan, et. al. "Mixed-Polar-Amplitude-Modulation Pilot-Based Blind Phase Estimation for Coherent Optical OFDM System", improved PBB phase estimation method named MPAM-PBB method for CO-OFDM has been proposed. A 16-QAM CO-OFDM optical B2B transmission simulation has been demonstrated to compare the performances of the proposed MPAM-PBB method with the conventional PAB method and the previous PBB method. The results show that the proposed MPAM-PBB method can achieve comparable performance with $\mathrm{PAB}$ and $\mathrm{PBB}$ method with the improved SE and non-increase of the computational complexity.

[3] Son Thai Le, et. al. "Blind Phase Noise Estimation for CO-OFDM Transmissions", concluded that blind PNE is efficiently practical for CO-OFDM communication with BPS and DDF blind PNE. Using only three test phases, DDF blind PNE method proposed performance as good as BPS with 16 tests phases, and thus, offering an effective solution for practical implementation. In addition, when the number of subcarriers is sufficient $(N>200)$ the difficulty of DDF blind was considerably reduced with two novel projected cost functions.

[4] Son Thai Le, et. al. "Quasi-Pilot Aided Phase Noise Estimation for Coherent Optical OFDM Systems", proposed a novel ordinary stage mistake evaluation method based on comparing the phase of pilot tendency with data subcarriers. Numerical simulation results confirmed that by setting PSs in correlation with data subcarriers the overhead of pilot give support to carrier phase assessment may be compact by a factor of 2 for two different correlation techniques, conjugated pilots and segment similarity pilots. In addition, in comparison with RF-pilot tone, the proposed methods offered a similar performance at the same overhead while extensively reducing the complexity in implementation.

[5] Mhatli Sofien, et. al. "Performances comparison of a coherent optical OFDM system", proposed estimation of the arrangement of a coherent optical OFDM modem by numerical simulations. They interpreted the effect of number of sub-carriers, elevated order modulations, data rate intended for the modem design in long distance up to $1400 \mathrm{~km}$.

Table 1 : Tabular summary of literature review

\begin{tabular}{|c|c|c|c|}
\hline $\begin{array}{l}\text { Citatio } \\
\text { n }\end{array}$ & Title & $\begin{array}{l}\text { Journal } \\
\text { Name/Year }\end{array}$ & Remark \\
\hline [1] & $\begin{array}{l}\text { Enhanced } \\
\text { Channel } \\
\text { Equalizers for } \\
\text { Adaptive } \\
\text { Zero-Guard- } \\
\text { Interval CO- } \\
\text { OFDM } \\
\text { Systems }\end{array}$ & $\begin{array}{l}\text { IEEE Photonics } \\
\text { Technology } \\
\text { Letters,2015 }\end{array}$ & $\begin{array}{l}\text { Transmission } \\
\text { distance is } \\
\text { increased } \\
\text { with FD- } \\
\text { BLMS and } \\
\text { FD-BRLS } \\
\text { adaptive } \\
\text { equalizers }\end{array}$ \\
\hline [2] & $\begin{array}{l}\text { Mixed-Polar- } \\
\text { Amplitude- } \\
\text { Modulation }\end{array}$ & $\begin{array}{l}\text { 14th } \\
\text { International } \\
\text { Conference on }\end{array}$ & $\begin{array}{l}\text { Proposed } \\
\text { MPAM-PBB } \\
\text { method }\end{array}$ \\
\hline
\end{tabular}

\begin{tabular}{|c|c|c|c|}
\hline & $\begin{array}{l}\text { Pilot-Based } \\
\text { Blind Phase } \\
\text { Estimation } \\
\text { for Coherent } \\
\text { Optical } \\
\text { OFDM } \\
\text { System }\end{array}$ & $\begin{array}{l}\text { Optical } \\
\text { Communication } \\
\text { s and Networks } \\
(\text { ICOCN }), 2015 .\end{array}$ & $\begin{array}{l}\text { achieves } \\
\text { improved SE } \\
\text { and same } \\
\text { computationa } \\
1 \text { complexity } \\
\text { as PAB and } \\
\text { PBB method. }\end{array}$ \\
\hline [3] & $\begin{array}{l}\text { Blind Phase } \\
\text { Noise } \\
\text { Estimation } \\
\text { for CO- } \\
\text { OFDM } \\
\text { Transmission } \\
\text { s }\end{array}$ & $\begin{array}{l}\text { Journal of Light } \\
\text { wave } \\
\text { Technology, } \\
2015\end{array}$ & 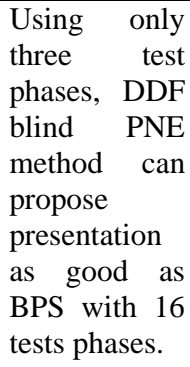 \\
\hline [4] & $\begin{array}{l}\text { Quasi-Pilot } \\
\text { Aided Phase } \\
\text { Noise } \\
\text { Estimation } \\
\text { for Coherent } \\
\text { Optical } \\
\text { OFDM } \\
\text { Systems }\end{array}$ & $\begin{array}{l}\text { IEEE Photonics } \\
\text { Technology } \\
\text { Letters, } \\
2014\end{array}$ & $\begin{array}{l}\text { A novel } \\
\text { ordinary } \\
\text { stage mistake } \\
\text { evaluation } \\
\text { method is } \\
\text { proposed } \\
\text { based on } \\
\text { comparing } \\
\text { the phase of } \\
\text { pilot } \\
\text { tendency } \\
\text { with data } \\
\text { subcarriers. }\end{array}$ \\
\hline [5] & $\begin{array}{l}\text { Performances } \\
\text { comparison } \\
\text { of a coherent } \\
\text { optical } \\
\text { OFDM } \\
\text { system }\end{array}$ & $\begin{array}{l}\text { Conference } \\
\text { Paper · march } \\
2014 .\end{array}$ & $\begin{array}{l}\text { It uses high } \\
\text { order } \\
\text { modulation } \\
\text { technique }\end{array}$ \\
\hline
\end{tabular}

\section{METHOD}

In this paper proposed the improvements in fiber nonlinear tolerance and channel estimation accuracy over conventional training symbol (TS)-based equalizers and data aided adaptive equalizers, in 16 quadrature amplitude modulation (QAM) CO-OFDM systems with artificial neural network(ANN). In this paper proposed modal show in figure in this first mapped onto various OFDM sub-carriers, which are then converted into time domain symbols by using IFFT After passing through a serial to parallel converter, the encoded complex data are first mapped onto various CO-OOFDM subcarriers. The inverse fast Fourier transform (IFFT) is then applied to convert the $\mathrm{N}$ number of sub-carriers into time-domain $\mathrm{CO}$ OOFDM symbols.

The $n$-th OFDM symbol is constructed by adding a CP of length $\mathrm{G}$ samples, which is added to $\mathrm{x}[\mathrm{n}]$, the parallel symbols of the IFFT, before being serialized to form a long digital complex data sequence. The real (I) and imaginary (Q) parts of the data sequence are separated and subsequently inputted into two DACs. The OFDM electrical-to-optical conversion is accomplished by feeding the OFDM I and Q components to the corresponding I/Q ports of the two optical MZMs. The optical modulators that have a sinusoidal transfer function are biased at the null biasing point; moreover, a 90-degree phase shifter is applied to the output of the second MZM. 


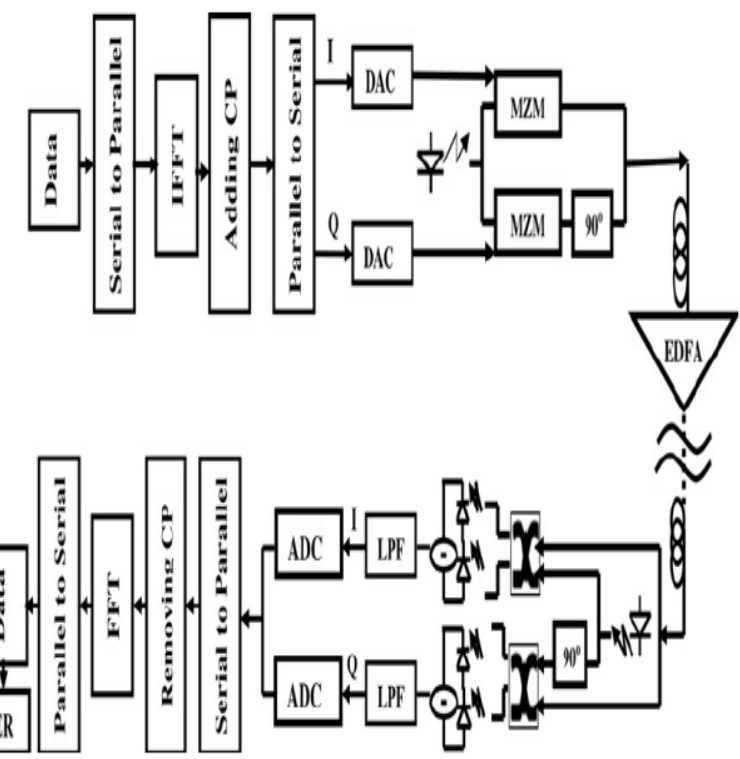

Fig.1 CO-OOFDM modem diagram used in numerical simulations

Finally, the optical signal is coupled to the SMF link of several spans with erbium-doped fiber amplifiers (EDFAs) inserted between them. At the receiver side, the COOOFDM signal is detected using two identical optical coherent balanced detectors acting as an optical- to-electrical OFDM I/Q converter, in which I/Q components of a locally generated carrier are mixed with the optical signal to obtain the electrical I/Q components. Each coherent detector consists of a pair of couplers and PIN detectors. The low-pass filters attenuate the sub- carriers secure to the Nyquist frequency, thus reducing the usable bandwidth as the attenuated sub-carriers cannot be used for the data transmission.

\section{CONCLUSION}

For CO-OOFDM modem, an ANN based equalization method with the RP learning algorithm was proposed proved to be more effective in combating $\mathrm{CD}$ when compared with the ANN equalizer with OSS learning algorithm and the traditional LMS equalization process.CO-OOFDM has been consider as a capable .procedure for future high-capacity optical networks, its practical application has been mainly determined by its tolerance to the optical fiber CD and susceptibility to the fiber nonlinearity particularly at high data rates and high optical power levels. Therefore, addressing these technical challenges and increasing CO- OOFDM system tolerance to $\mathrm{CD}$ and fiber non-linearities was the main focus of our proposed work, for which, a comprehensive investigation have been undertaken in order to investigate the feasibility of the OOFDM technique in coherent optical transmission systems with and without the aid of artificial neural network equalizer.

\section{REFERENCES}

[1] Wei Wang, Qunbi Zhuge, Yuliang Gao, Meng Qiu, Mathieu Chagnon, Mohammed Y. Sowailem,Fangyuan Zhang, and David V. Plant,et. al. "Enhanced Channel Equalizers for Adaptive Zero-Guard-Interval CO-OFDM Systems" IEEE Photonics Technology Letters, vol. 27, no. 16, August 15, 2015.

[2] Man Pan, Xuebing Zhang, J ianping Li and Zhaohui Li,et. al. "Mixed-Polar-Amplitude-Modulation PilotBased Blind Phase Estimation for Coherent Optical OFDM System", 14th International Conference on Optical Communications and Networks (ICOCN) Nanjing, China, 2015.

[3] Son Thai Le, Paul A. Haigh, Andrew D. Ellis and Sergei K. Turitsyn,et. al. "Blind Phase Noise Estimation for CO-OFDM Transmissions", Journal of Lightwave Technology, 2015.

[4] Son Thai Le, Thavamaran Kanesan, Elias Giacoumidis, Nick J. Doran, and Andrew D. Ellis, et. al. "Quasi-Pilot Aided Phase Noise Estimation for Coherent Optical OFDM Systems", IEEE Photonics Technology Letters, vol. 26, no. 5, March 1, 2014.

[5] Sofien Mhatli, Mutsam.A.Jarajreh, Bechir nsiri, Rabah Attia,et. al. "Performances comparison of a coherent optical OFDM system”, Conference Paper • march 2014.

[6] Changyuan YU, Pooi-Yuen KAM1, Shengjiao CAO,et. al. "Carrier recovery in coherent receiver of optical orthogonal frequency division multiplexing system", Higher Education Press and Springer-Verlag Berlin Heidelberg 2014

[7] W. Wang et al., "Low overhead and nonlinear-tolerant adaptive zeroguard- interval CO-OFDM," Opt. Exp., vol. 22, no. 15, pp. 17810-17822,Jul. 2014.

[8] J. G. Proakis, Digital Communications, 5th ed. New York, NY, USA: McGraw-Hill, 2007, pp. 640-668.

[9] W. Wang, Q. Zhuge, Y. Gao, and D. Plant, "Design of enhanced channel equalizers for adaptive zero-guardinterval CO-OFDM systems,"'in Proc. OFC, 2015, paper Th2A.26.

[10] S. Haykin, Adaptive Filter Theory, 3rd ed. Upper Saddle River, NJ, USA: Prentice-Hall, 1996, pp. 365-588.

[11] Q. Zhuge, M. H. Morsy-Osman, and D. V. Plant, "Low overhead intrasymbol carrier phase recovery for reducedguard-interval CO-OFDM,'J. Lightw. Technol., vol. 31, no. 8, pp. 1158-1169, Apr. 15, 2013. 